\title{
Improving the efficiency of discharge summary completion by linking to preexisiting patient information databases
}

\author{
Samuel Chan, Andrew P Maurice, Clifford W Pollard, Stephen J Ayre, Darren L Walters, Helen E Ward \\ The Prince Charles Hospital, Brisbane, Queensland, Australia
}

\begin{abstract}
The discharge summary (DS) is a document that contains the diagnosis, comorbidities, procedures, complications, and future treatment plan for a particular patient after an inpatient hospital stay. The DS is completed by junior medical staff and is delivered to the general practitioner (GP). DS completion is time consuming and tedious, and DSs are usually not completed within the recommended time frame after a patient is discharged. Time spent completing DSs correlate to junior doctor overtime, which costs the hospital money in overtime pay.

Information that is required in the DS is generally already entered into numerous electronic information systems in the hospital, including the "electronic patient journey board" which lists all the patients in a given ward with their clinical information. This information is constantly updated by all staff in the hospital. A program was developed that transferred this information directly into the patient DS. Ten junior doctors in two departments kept daily records for one week of the time spent compiling DSs, the time at work and the actual overtime claimed, before and after the introduction of the intervention.
\end{abstract}

The mean $( \pm S D)$ time for DS compilation per week reduced by $2.8( \pm 2.4)$ hours from $10.0( \pm 3.5)$ hours $(p<0.01)$ and the mean overtime worked per week reduced by $2.8( \pm 3.1)$ hours from $8.5( \pm 4.4)$ hours $(p<0.05)$. The mean overtime claimed reduced by $1.8( \pm 2.8)$ hours from $5.3( \pm 5.4)$ hours per week $(p<0.05)$, resulting in reduction in mean overtime payment of $\$ 114.95$ from $\$ 290.57$ per doctor, per week. Extrapolating to the 60 ward based junior doctors, the potential annual savings for the hospital budget are over $\$ 350,000$. Additionally, the number of DSs completed within 48 hours increased from $45 \%$ to $58 \%$.

In summary, the transfer of electronic data from the electronic patient journey board to the discharge summary program has yielded improvements in DS completion rates and overtime worked by medical staff, resulting in significant reduction in overtime costs.

\section{Problem}

At The Prince Charles Hospital (TPCH), the discharge summary (DS) is usually the only means of communicating the inpatient treatment summary and future plan to the general practitioner (GP).(1-3) At our institution, DSs are authored by resident medical officers (RMO), who are junior medical staff, on behalf of the treating consultant. The DS is forwarded to the patient's GP. DSs are created by electronic means at our institution using the "electronic discharge summary program" (EDSP). When a DS is completed, the information is sent electronically $(80 \%)$ or by post to the GP.

The DS plays a critical role in ensuring safe and effective continuity of care for patients being discharged from hospital.(4-7) Timely preparation of patient DSs have been associated with decreased rates of readmission.(8)

Our institution recommends that DSs are ideally completed at the time of patient discharge and no later than 48 hours after discharge. DSs are often not completed for several days or even weeks after a patient is discharged from hospital.

Many patients are encouraged to see their GP within one week postdischarge from hospital, either for medication supply, for investigations such as repeat blood tests or for a review of their clinical status. As a consequence of the lack of timely supply of the DS, the GP will often have minimal information regarding the patient's admission and lack guidance on what further clinical care is required. For example, a patient may have sustained an acute kidney injury in hospital and require serial urea and electrolyte blood tests. If the GP does not know this, there is potential for significant patient harm.

\section{Background}

TPCH is a 600 bed tertiary public teaching hospital in Brisbane, Queensland, Australia, and provides services in the Heart and Lung Institute (cardio-thoracic medicine and surgery), general medicine, general surgery, orthopaedics, rehabilitation and acute stroke, geriatrics, palliative care and mental health. TPCH has approximately 3500 staff of which there are 277 junior and 143 senior medical staff.

At TPCH, over 11,000 discharge summaries were completed by RMOs in the year 2013. Thus, improving the speed of DS completion by a few minutes per DS will potentially have large time saving implications.

At our institution two major factors contribute to untimely and 
inefficient DS completion:

- Time and workforce

Workload impacts on the capacity of RMOs to author DSs in a timely and efficient manner. RMOs are under considerable time pressure to complete the tasks expected of them. Their working hours are long (9) and many feel their load is not fairly or equitably distributed.(10) When the RMO is busy, "front line" medical care takes precedence such as ward work, caring for acutely unwell patients, communicating, and documenting team decisions. $(11,12)$ The literature has established that many RMOs are interrupted frequently in the course of their daily work and are commonly required to multi-task $(12,13)$, which also creates difficulty in authoring an accurate DS. As a consequence of multiple competing interests, the completion of DSs is a low priority for RMOs. Anecdotally, many RMOs at our institution have found it difficult to complete DSs within 48 hours of discharge. Many RMOs have also indicated that they do not have the capacity to complete the documents within rostered hours, which is usually eight hours of paid working time each day.

\section{- Duplication of data}

Completion of the DS involves duplication of data that has already been entered into other documents during the patient's admission. Hence, many RMOs have found it frustrating that information must be written repeatedly, and time could be saved if there was a mechanism to reduce duplication.

\section{Baseline measurement}

RMOs were trained in the use of the EDSP at the commencement of the academic year. In March 2013, after two months of using the system, five RMOs from thoracic medicine and five RMOs from internal medicine at TPCH were surveyed over one week. Each RMO was provided with a time sheet to document start and finish times each day, hours of overtime claimed, as well as any time undertaking work in the EDSP (Figure 1). The cost of overtime claimed was calculated from the average salary of a resident medical officer, which at the time was Australian $\$ 31.90$ per hour. The time after patient discharge of each completed DS was obtained from the EDSP for each doctor for the study week. At the end of the study week, the ten RMOs each provided their personal time sheet to the study authors for analysis.

From this data, four key metrics were calculated:

- The time spent working on discharge summaries

- The time RMOs worked from which hours of overtime was calculated

- The hours of overtime the RMO claimed (not necessarily the same as the true number of hours worked)

- The proportion of DSs completed within forty-eight hours of patient discharge.

The change in the PEJB took place in August, 2013. RMOs from internal medicine and thoracic medicine were given resource sessions and presentations by the study authors to learn about the program approximately four months prior to re-evaluation. RMOs from the same 10 units in thoracic medicine and internal medicine were surveyed in December 2013 for a one week period to evaluate the four metrics previously collected. Again, RMOs from these units recorded their personal time on a sheet provided to them and this was collected at the end of the week. This information was then entered onto a Microsoft Excel spreadsheet.

It is important to note that RMOs rotate across five different clinical specialities throughout the year. Hence, the RMOs surveyed in March, 2013 were different from the RMOs surveyed in December, 2013.

See supplementary file: ds3402.doc - "Figure 1"

\section{Design}

Each ward at TPCH utilises the patient electronic journey board (PEJB) (Figure 2). The PEJB contains an up to date list of all the patients on the ward and extensive patient information including the principal diagnosis, co-morbidities, procedures performed, complications, allied health information, and the treatment plan (Figure 3). Information regarding discharge planning is also a key part of the PEJB. This information is entered by all clinicians in the hospital when the patient is first admitted, and is updated on a regular basis as the clinical status of the patient changes.

The PEJB has been a successful tool for the medical, nursing and allied health teams to co-ordinate patient care, as it provides a succinct summary of the current issues affecting patient stay. Additionally, the PEJB is used to form printed "patient lists" for clinicians to use on medical ward rounds and to keep track of patients throughout the day. The information in the PEJB is very similar to the information that is required in the DS.

The intervention implemented in this QI study was the development of a computerised system to allow automatic transfer of patient information from the PEJB into the DS. The DS would still be manually checked for errors and amended as necessary after transfer of data.

Discharge medications were reviewed and entered into the DS by the pharmacist and the RMO, and reconciled by the pharmacist before inclusion in the DS. This process did not change. The completed DS was then sent to the GP (Figure 4).

The change in the PEJB took place in August, 2013. RMOs from internal medicine and thoracic medicine were given resource sessions and presentations by the study authors to learn about the program approximately four months prior to re-evaluation. RMOs from the same 10 units in thoracic medicine and internal medicine were surveyed in December 2013 for a one week period to evaluate the four metrics previously collected. Again, RMOs from these units recorded their personal time on a sheet provided to them and this was collected at the conclusion of the post-measurement study. This information was then entered onto a Microsoft Excel spreadsheet. 


\section{Strategy}

The authors worked with the Business Solutions Unit at TPCH to develop an electronic solution to transfer information from the PEJB to the patient's DS. The system cost approximately $\$ 40,000$ to develop and implement. Ideas were presented at departmental and hospital wide-forums, and at a national Quality Improvement Forum. Junior doctors trialled the process and provided feedback. The authors informed staff electronically regarding implementation of this project. Orientation days for medical staff regarding the program were provided. The hospital executive director and the hospital executive director of medical services and other senior hospital medical staff sponsored this project and assisted in implementing the system.

\section{Results}

DS metrics before and after the intervention are given in Table 1, 2, and 3. Comparisons before and after the intervention were made using the paired t-test.

At baseline, the mean time RMOs spent compiling discharge summaries varied from 2.5 hours to 14.25 hours weekly, which was similar to the mean overtime RMOs worked each day (between 1.5 hours to 13.5 hours). The mean overtime claimed also varied considerably from 0 hours to 14.25 hours weekly.

After the PEJB link was introduced, the mean $( \pm$ SD) time for DS work per week reduced by $2.8( \pm 2.4)$ hours from $10.0( \pm 3.5)$ hours (paired $t$ test $p<0.01$ ) (Figure 5). The mean actual overtime per week reduced by $2.8( \pm 3.1)$ hours from $8.5( \pm 4.4)$ hours $(p<0.05)$, and the mean of overtime claimed reduced by $1.8( \pm 2.8)$ hours from $5.3( \pm 5.4)$ hours per week $(p<0.05)$.

The minimum RMO wage in 2013 was Australian $\$ 31.90$ per hour at our institution. Claimed overtime is paid at one and a half times that rate for the first 3 hours and two times that rate for each hour thereafter. Thus, payment for overtime across the 10 RMOs reduced by a mean of $\$ 114.95$ (from $\$ 290.57$ to $\$ 175.62$ ) per doctor, per week. At TPCH, there are approximately $60 \mathrm{RMOs}$ working in the wards where DSs are required. Thus, the potential annual savings for the hospital budget are over $\$ 350,000$.

The number of discharge summaries completed within 48 hours increased from $45 \%$ to $58 \%$. The number of discharge summaries completed within 30 days (well after the recommended time frame) increased from $92 \%$ to $98 \%$ (Table 1).

See supplementary file: ds3403.doc - "TABLES AND FIGURES"

\section{Lessons and limitations}

- At TPCH, we have found that copying pre-entered data from pre-existing patient databases was associated with a reduced time to work on DSs. Any strategy that reduces the time taken to complete a DS, even if it saves only a few minutes per DS, has substantial time saving implications given the large number of DSs an RMO must complete over a year

- Benefits of reduction in DS completion time include reduced overtime worked by RMOs and decreased financial implications for the hospital of paid overtime

- In most hospitals, a significant amount of clinical data is already entered into various programs such as electronic patient lists. Use of this data reduced the time spent completing DSs and may also lead to an improvement in the quality and accuracy of the DS. We believe use of our electronic system will improve the quality of the DS and this will be examined in a future project

- A possible confounding factor which may influence the interpretation of these results is the improvement in skill and experience from the RMOs over the eight months between measurements

\section{Conclusion}

The discharge summary is an important clinical document that summarises the inpatient care and future treatment plan and instructions for each patient and is delivered to the general practitioner. The discharge summary is time consuming, frequently not completed within recommended time frames, and represents a large duplication of work for junior medical staff. The discharge summary can be completed using pre-existing electronic databases that already contain substantial clinical information, reducing the time spent in compiling them, thus reducing overtime payments, and increasing the proportion that are completed in a timely manner.

\section{References}

1. Myers JS et al. Are discharge summaries teachable? The effect of a discharge summary curriculum on the quality of discharge summaries in an internal medicine residency program. Acad Med 2006; 81:S5-8.

2. McMilan TE, Allan W, Black PN. Accuracy of information on medicines in hospital discharge summaries. Intern Med J 2006; 36:221-5.

3. Callen J, McIntosh J, Li J. Accuracy of medication documentation in hospital discharge summaries: A retrospective analysis of medication transcription errors in manual and electronic discharge summaries. Int $\mathrm{J}$ Med Inform 79: 58-64.

4. Bolton P. A quality assurance activity to improve discharge communication with general practice. J Qual Clin Pract $2001 ; 21: 69-70$.

5. Bolton P. A review of the role of information technology in discharge communications in Australia. Aust Health Rev 1999; 22:58-64.

6. Chow KM and Szeto CC. Secular trends in the medical discharge summary in an acute medical hospital. Postgrad Med J 2006; 82:615-8.

7. O'Leary KJ et al. Outpatient physicians' satisfaction with discharge summaries and perceived need for an electronic discharge summary. J Hosp Med 2006; 1:317-20. 


\section{BMJ Quality Improvement Reports}

8. Van Walraven C. Effect of discharge summary availability during post-discharge visits on hospital readmission. J Gen Intern Med 2002; 17:186-92.

9. AMA Safe Hours Audit 2006. 2006, Australian Medical Association.

10. AMA Junior Doctor Training, Education and Supervision Survey: Report of Findings. 2009, Australian Medical Association.

11. Westbrook Jl et al. All in a day's work: an observational study to quantify how and with whom doctors on hospital wards spend their time. Med J Aust 2008; 188:506-9.

12. Zhu JN et al. An observational study of emergency department intern activities. Med J Aust 2008; 189:514-9.

13. Volpp KG and Grande D. Residents' suggestions for reducing errors in teaching hospitals. N Engl J Med 2003; 348:851-5.

\section{Declaration of interests}

None declared

\section{Acknowledgements}

Nil 